\title{
Neurotoxicity of heroin-cocaine combinations in rat cortical neurons
}

\author{
Teresa Cunha-Oliveira ${ }^{a}$, A. Cristina Rego ${ }^{a, b}$, Jorge Garrido $^{d}$, Fernanda Borges ${ }^{e}$, Tice Macedo $^{c}$, \\ Catarina R. Oliveira ${ }^{\mathrm{a}, \mathrm{b}, *}$ \\ a Center for Neuroscience and Cell Biology of Coimbra, University of Coimbra, 3004-517 Coimbra, Portugal \\ ${ }^{\mathrm{b}}$ Faculty of Medicine, University of Coimbra, 3004-504 Coimbra, Portugal

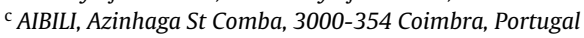 \\ d CIQUP, Chemical Engineering Department, School of Engineering, ISEP, Polytechnic Institute of Porto, 4200-072 Porto, Portugal \\ e CIQUP, Department of Chemistry, Faculty of Sciences, University of Porto, 4169-007 Porto, Portugal
}

\section{A R T I C L E I N F O}

\section{Article history:}

Received 4 May 2010

Received in revised form 22 June 2010

Accepted 23 June 2010

Available online 30 June 2010

\section{Keywords:}

Cocaine

Heroin

Speedball

Cortical neurons

Neurotoxicity

Apoptosis

Necrosis

\begin{abstract}
A B S T R A C T
Cocaine and heroin are frequently co-abused by humans, in a combination known as speedball. Recently, chemical interactions between heroin (Her) or its metabolite morphine (Mor) and cocaine (Coc) were described, resulting in the formation of strong adducts. In this work, we evaluated whether combinations of Coc and Her affect the neurotoxicity of these drugs, using rat cortical neurons incubated with Coc, Her, Her followed by $\mathrm{Coc}(\mathrm{Her}+\mathrm{Coc})$ and Her plus $\mathrm{Coc}(\mathrm{Her}: \mathrm{Coc}, 1: 1)$. Neurons exposed to Her, Her + Coc and Her:Coc exhibited a decrease in cell viability, which was more pronounced in neurons exposed to Her and $\mathrm{Her}+\mathrm{Coc}$, in comparison with neurons exposed to the mixture (Her:Coc). Cells exposed to the mixture showed increased intracellular calcium and mitochondrial dysfunction, as determined by a decrease in intracellular ATP levels and in mitochondrial membrane potential, displaying both apoptotic and necrotic characteristics. Conversely, a major increase in cytochrome c release, caspase 3-dependent apoptosis, and decreased metabolic neuronal viability were observed upon sequential exposure to Her and Coc. The data show that drug combinations potentiate cortical neurotoxicity and that the mode of co-exposure changes cellular death pathways activated by the drugs, strongly suggesting that chemical interactions occurring in Her:Coc, such as adduct formation, shift cell death mechanisms towards necrosis. Since impairment of the prefrontal cortex is involved in the loss of impulse control observed in drug addicts, the data presented here may contribute to explain the increase in treatment failure observed in speedball abusers.
\end{abstract}

(c) 2010 Elsevier Ireland Ltd. All rights reserved.

\section{Introduction}

Heroin and cocaine are two of the most harmful drugs of abuse known (Nutt et al., 2007) and their combination (speedball) is still very common in drug users entering treatment in Europe (EMCDDA, 2009). The popularity of this drug association may be explained by the complementary effects of the drugs, since cocaine is a psychostimulant, whereas heroin is a sedative.

According to the European Monitoring Center for Drugs and Drug Addiction (EMCDDA, 2008), treatment of polyabusers of cocaine and heroin is becoming more and more important, since cocaine is increasingly used by current or former heroin users (EMCDDA, 2008), sequentially and/or simultaneously (Leri et al.,

\footnotetext{
* Corresponding author at: Center for Neuroscience and Cell Biology of Coimbra, Faculty of Medicine, Rua Larga, University of Coimbra, 3004-504 Coimbra, Portugal. Tel.: +351239 820190; fax: +351239822776.

E-mail addresses: catarina.n.oliveira@gmail.com, catarina@cnc.cj.uc.pt (C.R. Oliveira).
}

2003). Moreover, the use of cocaine in combination with heroin is associated with the presence of a mental illness and may aggravate underlying psychological problems such as bipolar disorder (EMCDDA, 2008). Thus, speedball abusers seem to exhibit a more severe psychopathology as compared to other cocaine addicts, and are more likely to fail in drug abuse treatment (Bandettini di Poggio et al., 2006).

We have previously demonstrated that both cocaine (CunhaOliveira et al., 2006) and heroin (Cunha-Oliveira et al., 2007) per se induce neurotoxicity in rat cortical neurons, upon an acute exposure, in a process involving mitochondrial dysfunction and cell death by apoptosis. However, despite the high prevalence and severe effects of speedball, little is known about the toxicity of this drug combination.

Cocaine and morphine were previously shown to interact at the molecular level, giving rise to a new chemical entity, a cocaine-morphine adduct (Garrido et al., 2007). Therefore, chemical interactions between cocaine, heroin or their metabolites, such as cocaine-morphine adducts, may play a role in the effects of cocaine-heroin combinations, especially when the co-abuse occurs simultaneously and chemical interactions most probably occur. 
The present study was designed to evaluate if chemical interactions between drugs may play a role in speedball neurotoxicity, using primary cultures of rat cortical neurons subjected to heroin and/or cocaine, administered alone, sequentially or simultaneously. We hypothesize that cocaine-heroin combinations are more toxic than the drugs alone and that the pathways of neurotoxicity caused by co-administration of cocaine and heroin (1:1) are distinct from those evoked by a sequential administration of these drugs, due to occurrence of chemical interactions between the two drugs (or their metabolites).

\section{Materials and methods}

\subsection{Materials}

Neurobasal medium and B27 supplement were purchased from Gibco (Paisley, UK). Ac-DEVD-pNA, resazurin, FCCP, Hoechst 33342, Propidium Iodide and anti-Microtubule-Associated Protein 2 (MAP-2) were supplied by Sigma-Aldrich (St Louis, MO, USA). Indo-1/AM, Tetramethyl Rhodamine Methyl Ester (TMRM ${ }^{+}$), Alexa anti-rabbit 488 and Alexa anti-mouse 594 were obtained from Invitrogen (San Diego, CA, USA). Anti-cytochrome c was purchased from BD Pharmingen (San Diego, CA, USA). Mouse anti-GAPDH and mouse anti-HSP-60 were obtained from Chemicon (Billerica, MA, USA). ECF and anti-mouse IgG-alkaline-phosphatase-bound antibodies were purchased from GE Healthcare (Piscataway, NJ, USA). Anti-active-caspase-3 was obtained from Cell Signaling (Beverly, MA, USA). Heroin hydrochloride was synthesized from morphine obtained from Uquipa (Lisbon, Portugal), as described elsewhere (Garrido et al., 2004). All other reagents were of analytical grade.

\subsection{Chemical analysis}

Heroin hydrochloride ( $>98 \%$, HPLC) was synthesized by the classic method of acetylation, using acetic anhydride and pyridine (Garrido et al., 2004) at room temperature. Cocaine hydrochloride $(\geq 98.5 \%$, HPLC) was acquired from Sigma-Aldrich (St Louis, MO, USA). HPLC analyses were conducted on an HPLC system (Merck/Hitachi-LaChrom, Tokyo, Japan) equipped with a diode array detector (DAD). The analytical column was a commercially pre-packed reverse phase (RP18) column $(250 \mathrm{~mm} \times 4.0 \mathrm{~mm}$ i.d., $5 \mu \mathrm{m})$. An isocratic elution was performed at a flow rate of $1.5 \mathrm{~mL} / \mathrm{min}$ and the absorbance was measured at $216 \mathrm{~nm}$. The analysis was carried out, at room temperature using a solution of $60 \% 10 \mathrm{mmol} / \mathrm{L}$ aqueous ammonium acetate ( $\mathrm{pH} 3$ ) plus $40 \%$ acetonitrile as mobile phase.

\subsection{Cell culture}

Rat cortical neurons were isolated and cultured as previously described (CunhaOliveira et al., 2007). Briefly, the frontal cortices of rat embryos (16-17 days) were dissected, dissociated, and the cells were resuspended in Neurobasal Medium supplemented with B27 supplement, $0.2 \mathrm{mM}$ glutamine, $0.1 \mathrm{mg} / \mathrm{mL}$ streptomycin and $100 \mathrm{U} / \mathrm{mL}$ penicillin, plated in poly-L-lysine $(1 \mathrm{mg} / \mathrm{mL})$ coated multiwells or coverslips, and cultivated for $5-6$ days at $37^{\circ} \mathrm{C}$ in $95 \%$ air $/ 5 \% \mathrm{CO}_{2}$.

\subsection{Drug exposure}

Based on our previous studies (Cunha-Oliveira et al., 2006, 2007), the cells were incubated with solutions of $500-1000 \mu \mathrm{M}$ heroin hydrochloride or $500-1000 \mu \mathrm{M}$ cocaine hydrochloride, for $24 \mathrm{~h}$, in culture medium.

To study speedball neurotoxicity, heroin $(300-1000 \mu \mathrm{M})$ and cocaine (300-1000 $\mu \mathrm{M})$ were either administered (1:1) sequentially from separate stock solutions $(\mathrm{Her}+\mathrm{Coc})$, or simultaneously from a solution containing both drugs at $250 \mathrm{mM}$, prepared from a mixture of cocaine and heroin in the solid forms (Her:Coc).

\subsection{Analysis of metabolic activity}

The cells were incubated with $10 \mu \mathrm{g} / \mathrm{mL}$ resazurin in Neurobasal medium, for $2 \mathrm{~h}$ at $37^{\circ} \mathrm{C}$. The extent of resazurin reduction, corresponding to metabolic cell viability, was determined by the absorbance of the reduced compound (at $570 \mathrm{~nm}$ ) minus the absorbance of resazurin (at $600 \mathrm{~nm}$ ), in a Spectramax Plus 384 microplate spectrophotometer (Molecular Devices, Sunnyvale, CA), and normalized as a percentage of the control.

\subsection{Quantification of intracellular calcium levels}

The cells were incubated with $3 \mu \mathrm{M}$ Indo- $1 / \mathrm{AM}$ in basal medium (in mmol/L: $132 \mathrm{NaCl}, 4 \mathrm{KCl}, 1.4 \mathrm{MgCl}_{2}, 6 \mathrm{Glucose}, 10 \mathrm{HEPES}, 1 \mathrm{CaCl}_{2}$ ) for $45 \mathrm{~min}$ at $37^{\circ} \mathrm{C}$, and further incubated for $15 \mathrm{~min}$ in basal medium in the absence of Indo-1/AM, to ensure a complete hydrolysis of the acetoxymethyl ester of Indo-1. Indo- 1 fluorescence $(F)$ was measured for 4 min with excitation at $335 \mathrm{~nm}$ and emission at $410 \mathrm{~nm}$, in a Spectramax Gemini EM microplate spectrofluorometer (Molecular Devices, Sunnyvale, CA). Internal calibration of intracellular calcium was performed by incubation with
$3 \mu \mathrm{M}$ ionomycin for $8 \mathrm{~min}\left(F_{\max }\right)$ followed by incubation with $3 \mathrm{mM} \mathrm{MnCl}$, for $4 \mathrm{~min}$ (autofluorescence - AF). The free intracellular $\mathrm{Ca}^{2+}$ concentration $\left(\left[\mathrm{Ca}^{2+}\right]_{\mathrm{i}}\right)$ was calculated by the expressions: $\left[\mathrm{Ca}^{2+}\right]_{\mathrm{i}}=\mathrm{Kd} \times\left(F-F_{\min }\right) /\left(F_{\max }-F\right)$, with $\mathrm{Kd}=250 \mathrm{nM}$ and $F_{\min }=\mathrm{AF}+1 / 12 \times\left(F_{\max }-\mathrm{AF}\right)$.

\subsection{Evaluation of mitochondrial membrane potential}

The cells were incubated with $300 \mathrm{nM} \mathrm{TMRM}^{+}$for $1 \mathrm{~h}$ and the fluorescence $(F)$ was measured for $5 \mathrm{~min}$ with excitation at $540 \mathrm{~nm}$ and emission at $590 \mathrm{~nm}$, in a Spectramax Gemini EM microplate spectrofluorometer (Molecular Devices, Sunnyvale, $\mathrm{CA}$ ). $\mathrm{TMRM}^{+}$is accumulated in the mitochondria due to the mitochondrial transmembrane potential. Complete mitochondrial depolarization and release of mitochondrially retained $\mathrm{TMRM}^{+}$were induced by incubation with $2 \mu \mathrm{M} \mathrm{FCCP} \mathrm{plus}$ $1 \mu \mathrm{g} / \mathrm{mL}$ oligomycin (to avoid ATP synthase reversal) for $5 \mathrm{~min}\left(F_{\max }\right)$. Released $\mathrm{TMRM}^{+}$was determined as $F_{\max } / F$, and normalized in percentage of the control.

\subsection{Measurement of caspase-3 activity}

The cells were harvested in lysis buffer [in mmol/L: 25 HEPES, $2 \mathrm{MgCl}_{2}, 1 \mathrm{EDTA}$ 1 EGTA, 2 dithiothreitol, 0.1 phenylmethylsulfonyl fluoride (PMSF) and $1 \mu \mathrm{g} / \mathrm{mL}$ of protease inhibitor cocktail (chymostatin, leupeptin, antipain and pepstatin A)]. The resulting extracts were frozen and thawed three times, centrifuged at $15,000 \times \mathrm{g}$ for $10 \mathrm{~min}\left(4^{\circ} \mathrm{C}\right)$, and the protein in the supernatant fraction was quantified by the BioRad (Coomassie brilliant blue G250) protein assay. The supernatants were tested for the activity of caspase-3, at $405 \mathrm{nM}$, after reaction with Ac-DEVD-pNA ( $100 \mu \mathrm{M}$, for $3 \mathrm{~h}$ at $37^{\circ} \mathrm{C}$ ). The results were normalized over the control absorbance value, using the same amount of protein $(25 \mu \mathrm{g} /$ assay $)$.

\subsection{Analysis of intracellular ATP levels}

The cells were treated as for the caspase- 3 activity assay. The supernatants were assayed for adenine nucleotide determination by separation by reverse-phase high-performance liquid chromatography and the absorbance was monitored at $254 \mathrm{~nm}$. Peak identity was determined by following the retention time of known standards. ADP or AMP levels were not detectable under our experimental conditions. The amount of ATP was determined by comparison with a standard curve $(12.5-400 \mathrm{pmol})$, normalized for the amount of protein and presented in percentage of the controls.

\subsection{Analysis of cytochrome $c$ by Western blotting}

Cell lysates were homogenized in sucrose buffer (in mmol/L: 250 sucrose, 20 HEPES, $10 \mathrm{KCl}, 1.5 \mathrm{MgCl}_{2}, 1 \mathrm{EDTA}, 1$ dithiothreitol and 0.1 PMSF) supplemented with $1 \mu \mathrm{g} / \mathrm{mL}$ of protease inhibitor cocktail. The mitochondrial fraction (P2) was separated by centrifugation at $500 \times \mathrm{g}$ for $12 \mathrm{~min}\left(4^{\circ} \mathrm{C}\right)$ followed by centrifugation of the resulting supernatant at $12,000 \times \mathrm{g}$ for $20 \mathrm{~min}\left(4^{\circ} \mathrm{C}\right)$. Cytosolic fraction (P3) was obtained upon protein precipitation of the resulting supernatant with $5 \%$ trichloroacetic acid, followed by centrifugation at $15,800 \times \mathrm{g}$ for $10 \mathrm{~min}$ and $\mathrm{pH}$ neutralization with $\mathrm{NaOH}$.

Proteins $(15 \mu \mathrm{g} / \mathrm{sample})$ were denatured and separated by sodium dodecyl sulfate-polyacrylamide gel electrophoresis (10\%), and then transferred to a PVDF membrane, which was then incubated with mouse anti-cytochrome c (1:500), mouse anti-GAPDH (1:2500) and mouse anti-HSP-60 (1:1000) antibodies. The secondary detection was performed using anti-mouse IgG-alkaline-phosphatasebound antibodies $(1: 20,000)$. The bands were developed with ECF, visualized in a VersaDoc imaging system (Bio-Rad, Hercules, CA) and quantified using the Quantity One software (Bio-Rad, Hercules, CA).

\subsection{Immunocytochemistry}

The cells were fixed in $3.7 \%$ formaldehyde, permeabilized in $0.2 \%$ Triton X-100 for $2 \mathrm{~min}$, and blocked in 3\% BSA for 30 min. Coverslips were incubated overnight with primary antibodies against the active form of caspase-3 (1:1000) and anti-MAP$2(1: 200)$, followed by $1 \mathrm{~h}$ incubation with the secondary antibodies Alexa anti-rabbit 488 and anti-mouse 594 (1:1000). The nuclei were stained by using Hoechst 33342 $(1 \mu \mathrm{g} / \mathrm{mL})$ and the cells were visualized in an epifluorescence microscope (Zeiss Axioscope), under $200 \times$ magnification. Cell viability was evaluated by following nuclear morphology. Active-caspase-3 positive cells were counted, and the results presented in \% of total cells in the field.

\subsection{Analysis of nuclear morphology and membrane integrity}

For the analysis of cell viability, nuclear morphology was evaluated upon incubation with Hoechst $33342(1 \mu \mathrm{g} / \mathrm{mL})$. Condensed and fragmented nuclei were scored as "dead cells", and nuclei presenting a normal shape were scored as "viable cells".

For the analysis of apoptosis and necrosis, coverslips were incubated with $1 \mu \mathrm{g} / \mathrm{mL}$ Hoechst and $4 \mu \mathrm{g} / \mathrm{mL}$ propidium iodide (PI) for $3 \mathrm{~min}$ and immediately photographed in an epifluorescence microscope. Viable, apoptotic and necrotic cells were scored ( 3 fields by coverslip) by following the nuclear morphology and PI permeability, and the data presented in percent of total cells in the field. 


\subsection{Statistical analysis}

Data are the mean \pm SEM from at least three independent experiments, performed in duplicate or triplicate. Statistical analysis was performed by one-way ANOVA with Tukey post hoc test or $t$-test, where referred $(p<0.05$ was considered significant).

\section{Results}

\subsection{Combination of cocaine and heroin potentiates neuronal cell} death

In order to study the neurotoxicity of heroin and cocaine, we used two different combinations of the drugs, sequential and simultaneous, common in cocaine-heroin co-users (Leri et al., 2003). Cortical neurons in culture were incubated with the drugs sequentially (heroin followed by cocaine, Her $+\mathrm{Coc}$ ) or simultaneously (from a 1:1 solution containing the two drugs, Her:Coc). In the sequential exposure we first incubated cells with heroin, immediately followed by cocaine incubation, since heroin addicts who also use cocaine represent the main set of co-abusers seeking treatment (EMCDDA, 2008).

To test our hypothesis that combinations of heroin and cocaine are neurotoxic, we incubated rat cortical neurons with increasing concentrations of cocaine, heroin or their combinations. Metabolic viability was determined by the resazurin reduction assay (Fig. 1A). Decreased metabolic viability was observed in the presence of $1 \mathrm{mM}$ heroin or with combinations with $1 \mathrm{mM}$ cocaine (Fig. $1 \mathrm{~A}$ ), whereas cocaine alone did not affect resazurin reduction. At this concentration, heroin induced a decrease in metabolic activity by about 18.5\% ( $p<0.05)$, whereas Her + Coc and Her:Coc decreased the activity by $25.3 \%(p<0.01)$ and $11.4 \%(p<0.001, t$-test $)$, respectively. In this assay, Her + Coc solution was slightly more toxic than Her:Coc, inducing a higher decrease in metabolic activity, by $13.9 \%(p<0.05)$, suggesting that chemical interactions occurring in the drug mixture may affect the neurotoxicity of the drug combination. We further determined the number of viable and dying cells by following the nuclear morphology with Hoechst (Fig. 1B). We observed that cocaine $(6.5 \%, p<0.05, t$-test) and heroin $(9.6 \%$, $p<0.05)$ alone induced a slight increase in the number of dying cells, whereas both Her + Coc and Her:Coc increased this number by about $23 \%(p<0.001)$, when compared with the control (nontreated cells), suggesting that combinations of heroin and cocaine are more neurotoxic than each drug alone. The increase in dying cells observed upon cocaine incubation (Fig. 1B) seems to be too low to be detected by the resazurin reduction assay (Fig. 1A).

\subsection{Simultaneous exposure to heroin and cocaine induces a higher degree of mitochondrial dysfunction}

Since heroin-cocaine combinations induce cell death (Fig. 1), we analyzed three intracellular parameters normally affected in dying cells, in order to dissect the intracellular mechanisms involved in the neurotoxicity of these drug combinations and shed light on the cell death pathways responsible for the observed neurotoxicity. We observed that cocaine induced an increase in intracellular calcium levels, by about $41.7 \%(p<0.001)$ (Fig. $2 \mathrm{~A})$, a decrease in intracellular ATP levels, by about $31.2 \%(p<0.01)$ (Fig. 2B) and a decrease in mitochondrial membrane potential by $5.3 \%(p<0.05)$ (Fig. $2 \mathrm{C})$, when compared to untreated cells. Heroin alone only induced a significant decrease in mitochondrial membrane potential, by about $5.1 \%(p<0.05)$ (Fig. 2C).

Her + Coc caused a 29.6\% $(p<0.01)$ increase in intracellular calcium levels (Fig. 2A) and a $10.1 \%(p<0.001)$ decrease in mitochondrial membrane potential (Fig. 2C), when compared to control conditions, whereas Her:Coc induced a higher increase in intracel- lular calcium levels $(66.5 \%, p<0.001)$, a decrease in intracellular ATP levels (by $28.2 \%, p<0.01$ ) and a major decrease in mitochondrial membrane potential (by $15.8 \%, p<0.001$ ), when compared to control conditions (Fig. 2).

When comparing the two types of heroin-cocaine combinations, Her:Coc induced an increase by $36.9 \%(p<0.001)$ in intracellular calcium levels (Fig. 2A) and a decrease by $5.8 \%$ $(p<0.05)$ in mitochondrial membrane potential (Fig. 2C), compared to cells sequentially exposed to Her + Coc. The effects of heroin and cocaine on mitochondrial membrane potential seem to be additive, as seen in Her + Coc (Fig. 2C). However, the decrease in mitochondrial potential was more pronounced in Her:Coc, compared to $\mathrm{Her}+\mathrm{Coc}$, suggesting that possible chemical interactions may interfere with mitochondrial membrane potential. Moreover, in contrast with the sequential exposure to heroin and cocaine, the simultaneous exposure also induced a decrease in intracellular ATP levels, compared to control conditions, suggesting a higher degree of mitochondrial dysfunction accompanied by a rise in intracellular $\mathrm{Ca}^{2+}$, upon simultaneous exposure to the two drugs.

\subsection{Combinations of heroin and cocaine induce apoptotic hallmarks}

Since drug combinations caused mitochondrial dysfunction (Fig. 2) and we have previously shown that cocaine (CunhaOliveira et al., 2006) and street heroin (Cunha-Oliveira et al., 2007) induced mitochondrial-dependent apoptosis, we further analysed cytochrome c release and caspase- 3 activity, upon exposure to the drugs alone or to their combinations (Fig. 3).

Cocaine or heroin alone did not significantly affect cytochrome c levels, whereas both combinations of heroin and cocaine induced a decrease in mitochondrial cytochrome c levels when compared to controls, by about $59 \%(p<0.001)$ in the presence of $\mathrm{Her}+\mathrm{Coc}$ and $25.3 \%(p<0.05)$ in the presence of Her:Coc. Thus, when comparing the two heroin-cocaine combinations, Her + Coc induced a larger decrease $(33.7 \%, p<0.01)$ in mitochondrial cytochrome $\mathrm{c}$ levels (Fig. 3A).

Furthermore, we observed that cocaine induced an increase in caspase- 3 activity (by about $63 \%, p<0.01, t$-test), whereas heroin increased the number of active-caspase- 3 positive cells by about $5.2 \%$ ( $p<0.05 \%$ ) (Fig. 3B), in comparison with the control, confirming that both drugs may activate this effector caspase. Differences between caspase- 3 activity in cell populations and the number of cells expressing active-caspase- 3 may be explained if there are different numbers of active-caspase- 3 molecules in each individual cell. Both caspase- 3 activity and the number of cells positive for active-caspase- 3 were enhanced by the two drug combinations tested (Fig. 3B). Her + Coc induced an increase by $10.6 \%(p<0.001)$ in the number of active-caspase- 3 positive cells and a $149.4 \%$ $(p<0.01 \%)$ increase in caspase- 3 activity, when compared to the control. The simultaneous exposure to heroin and cocaine induced a $5.6 \%(p<0.05)$ increase in the number of active-caspase-3 positive cells, and a $103.1 \%(p<0.05)$ increase in caspase-3 activity, when compared to the control. These data suggest that different combinations of cocaine and heroin have distinguishable neurotoxic profiles.

\subsection{Simultaneous exposure to cocaine and heroin shifts cell death mechanisms towards necrosis}

In order to further characterize the cell death mechanisms involved in the toxicity of heroin and cocaine combinations, we quantified the percentage of apoptotic versus necrotic cells following their nuclear morphology and the permeability to propidium iodide (PI) (Fig. 4). We observed that both drug combinations induced an increase in the number of apoptotic cells ( $\mathrm{Her}+\mathrm{Coc}$ 

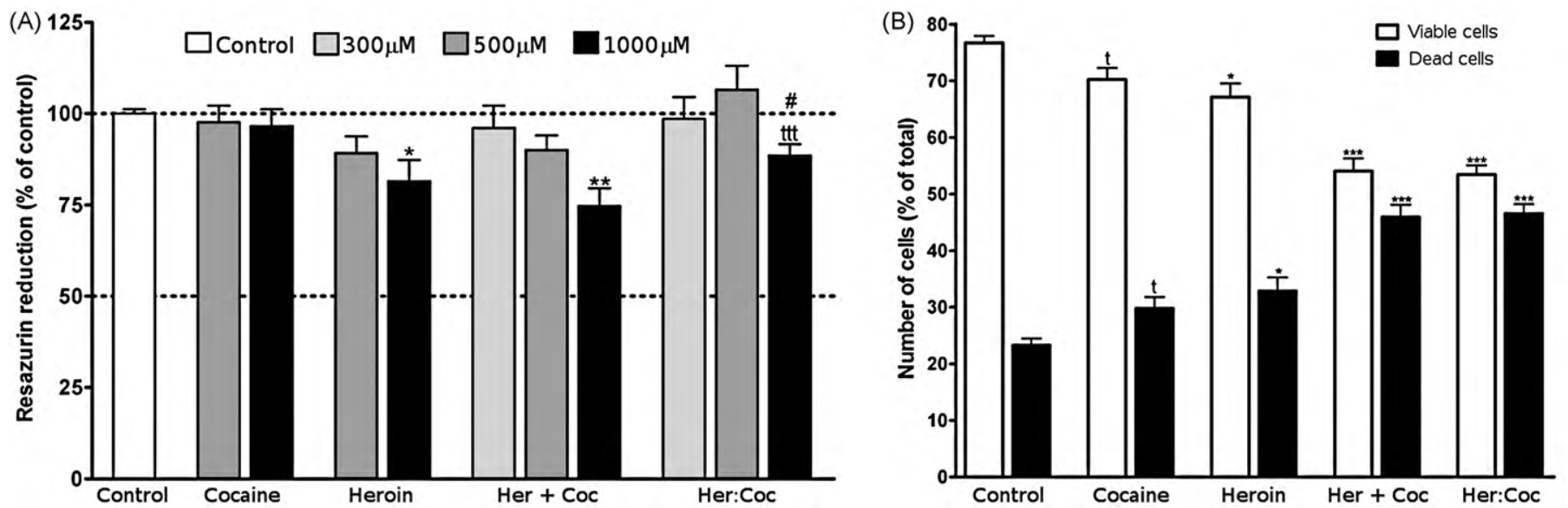

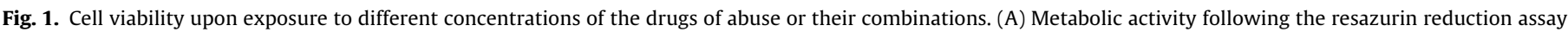

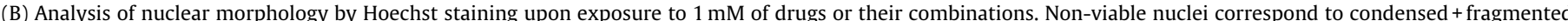

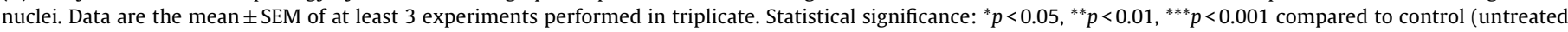

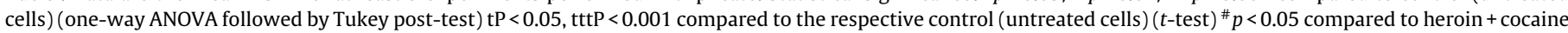
( $t$-test).

- 16.1\%, $p<0.001$; Her:Coc - 9.8\%, $p<0.001$ ) when compared to the control. In contrast, no significant effects were observed with cocaine or heroin alone. The simultaneous combination (Her:Coc) induced an increase $(9.8 \%, p<0.001)$ in the number of cells permeable to PI (necrotic cells), when compared to the control, whereas $\mathrm{Her}+\mathrm{Coc}$ did not elicit a significant number of necrotic cells. Therefore, the number of Her:Coc-induced necrotic cells was significantly higher when compared to other experimental conditions, suggesting that simultaneous exposure to cocaine and heroin (Her:Coc) shifts cell death mechanisms towards necrosis. The results are summarized in Fig. 5.

\section{Discussion}

In this study we demonstrated that combinations of cocaine and heroin are more neurotoxic than each drug alone, and that there are differences in the neurotoxic pathways elicited by sequential (Her $+\mathrm{Coc}$ ) or simultaneous (Her:Coc) exposure to both drugs (Fig. 5). Indeed, $\mathrm{Her}+\mathrm{Coc}$ elicited a major decrease in metabolic viability and an increase in cytochrome c release and caspase3 activation leading to apoptosis, whereas Her:Coc also caused necrotic cell death, most likely as a consequence of increased intracellular calcium, decreased mitochondrial membrane potential and decreased ATP levels (Orrenius et al., 2003).
The concentrations of drugs used in this study were chosen based on previous neurotoxicological data obtained in our lab (Cunha-Oliveira et al., 2006, 2007; Oliveira et al., 2002), and more importantly because in this concentration range a strong chemical drug-drug interaction was shown to occur in cocaine-heroin mixtures (Garrido et al., 2007), enabling the study of biological effects of these drug interactions.

Heroin metabolism occurs readily upon self-administration, by deacetylation to the active metabolites 6-monoacetylmorphine (6MAM) and morphine (Inturrisi et al., 1983). It was previously shown that, upon in vitro incubation of brain membranes with heroin, 38\% is recovered as heroin, $60 \%$ as 6-MAM and $2 \%$ as morphine (Selley et al., 2001). Indeed, the concentration of 6-MAM in the brain is higher than morphine's, upon exposure to heroin in humans (Goldberger et al., 1994; Rook et al., 2006), rats (Strandberg et al., 2006) and mice (Guillot et al., 2007). Moreover, the locomotor activity response upon heroin injection is mediated by 6-MAM (Andersen et al., 2009), with 6-MAM being more active at the $\mu$-opioid receptor, compared to morphine (Selley et al., 2001). Since heroin is not directly an opioid receptor agonist, it is thought to act mainly through its metabolites (Selley et al., 2001), and its effects on brain opioid receptors seem to be mainly mediated by 6-MAM. Mechanisms involving opioid receptors are possibly present under our conditions, since primary cortical neurons express $\mu$ - and $\delta$-opioid receptors (Lee et al., 2002). Besides the effects mediated by opi-
(A)

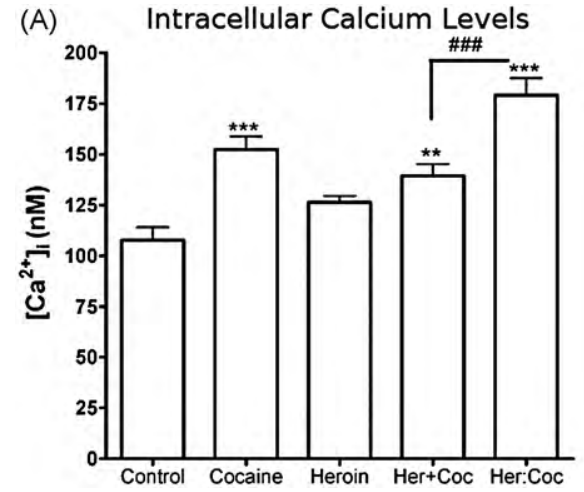

(B)

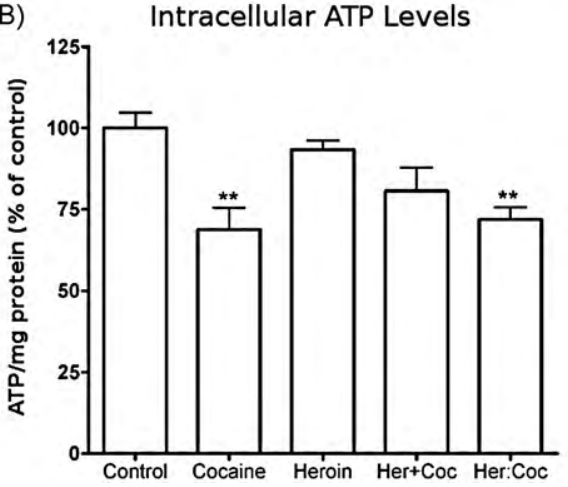

(C) Mitochondrial Membrane Potential

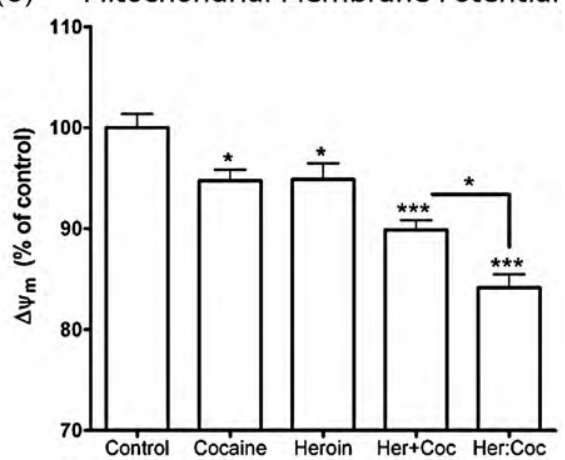

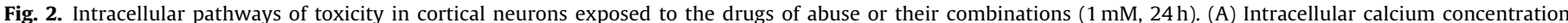

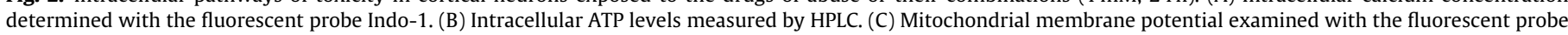

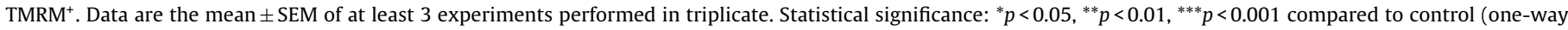
ANOVA followed by Tukey post-test) ${ }^{\# \# \# ~} p<0.001$ compared to heroin + cocaine (one-way ANOVA followed by Tukey post-test). 
(A)
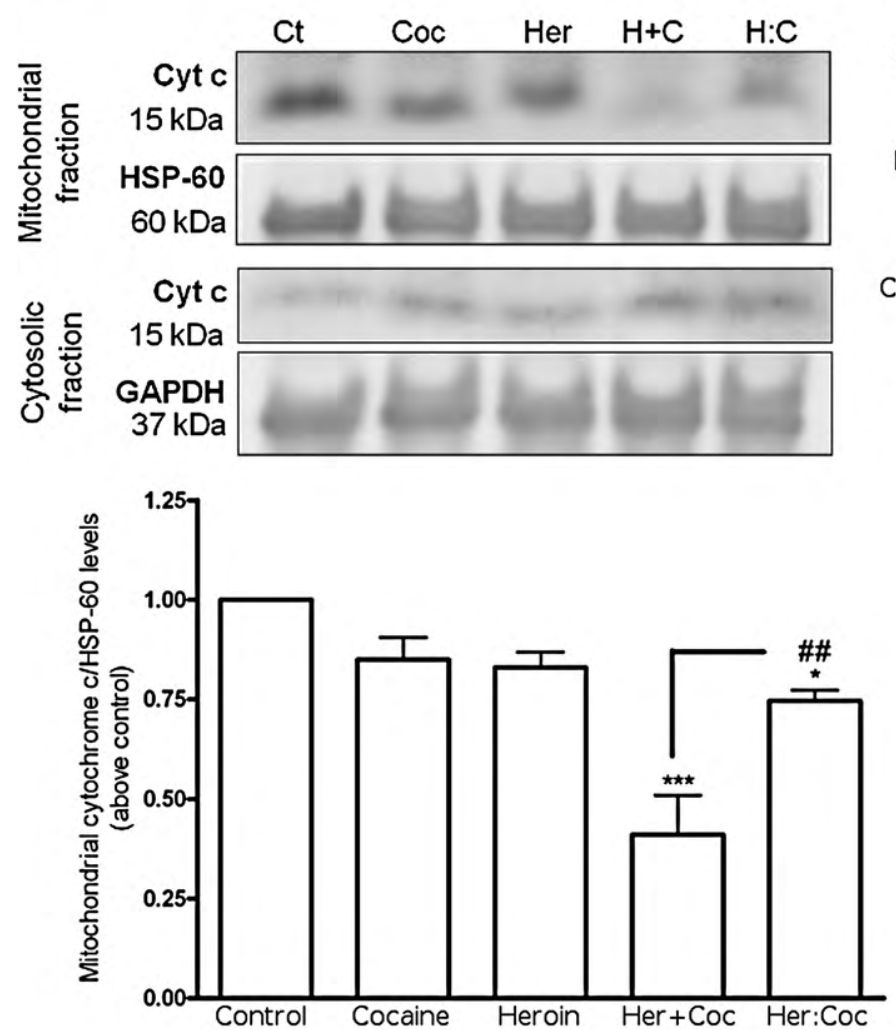

(B)

Overlay
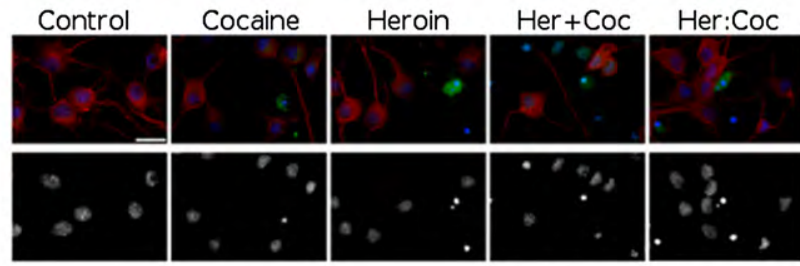

Active

Caspase- 3

MAP2
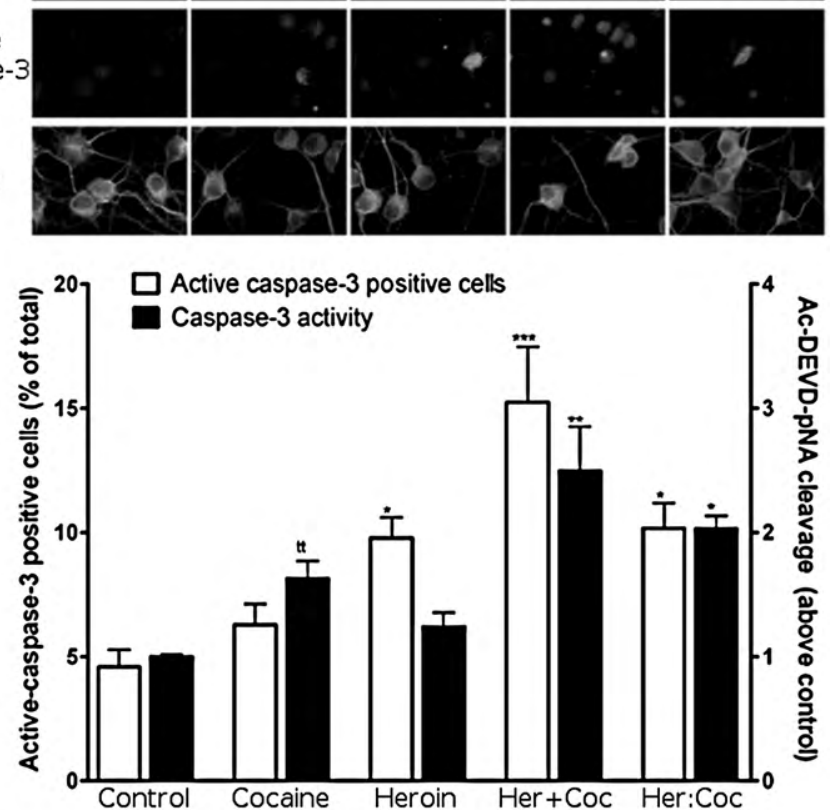

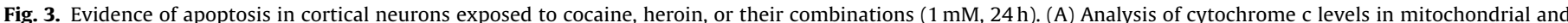

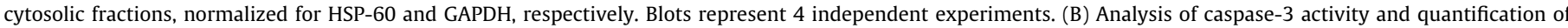

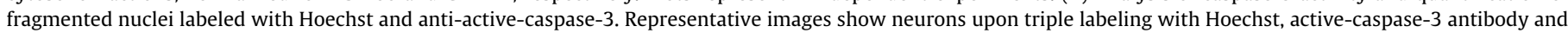

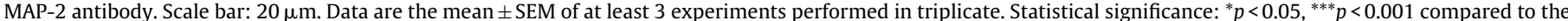

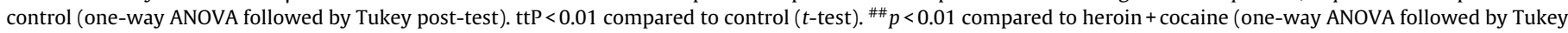
post-test).

oid receptors, it is also possible that heroin, due to its lipophilicity, enters into the cells to interact directly with intracellular targets. This hypothesis could explain the opioid receptor-independent mitochondrial dysfunction and apoptosis observed upon exposure to street heroin, in cortical neurons (Cunha-Oliveira et al., 2007). Similarly, taking into account the data obtained so far, in the present work the mechanism of heroin-induced decrease in metabolic activity and mitochondrial membrane potential is possibly independent of opioid receptors.

Cocaine plasma concentrations are usually on the low micromolar range, in human abusers, and in overdose cases plasma concentration of this drug may exceed $1 \mathrm{mM}$ (Heard et al., 2008; Yuan and Acosta, 2000). Cocaine crosses the blood-brain barrier and accumulates within the CNS and its brain concentrations after
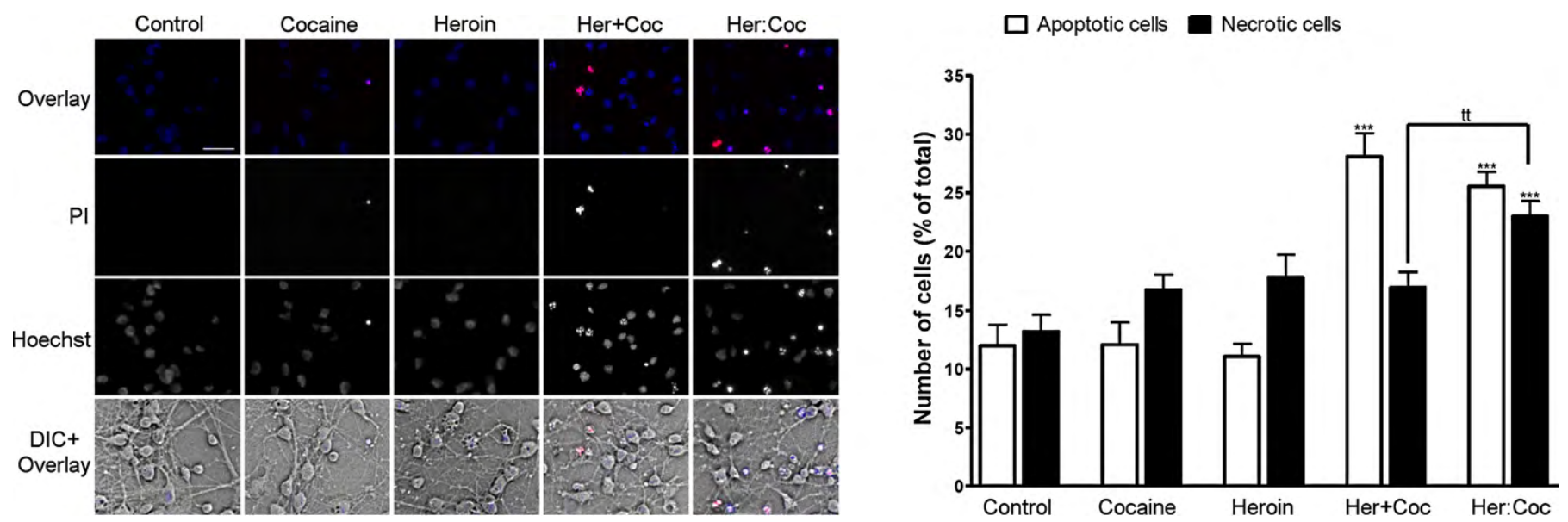

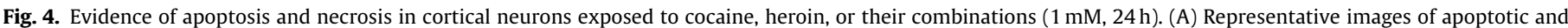

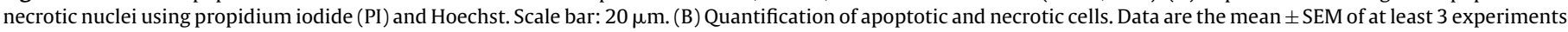

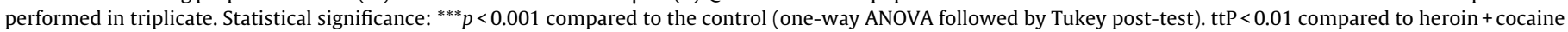
(t-test). 


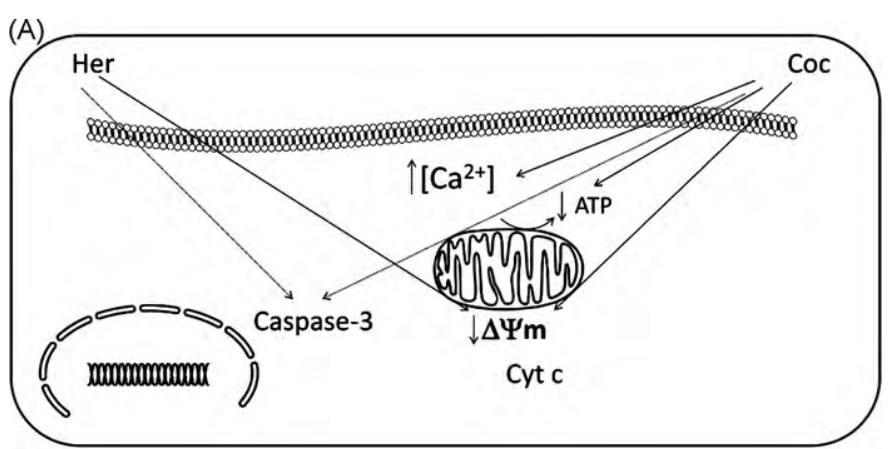

(B)

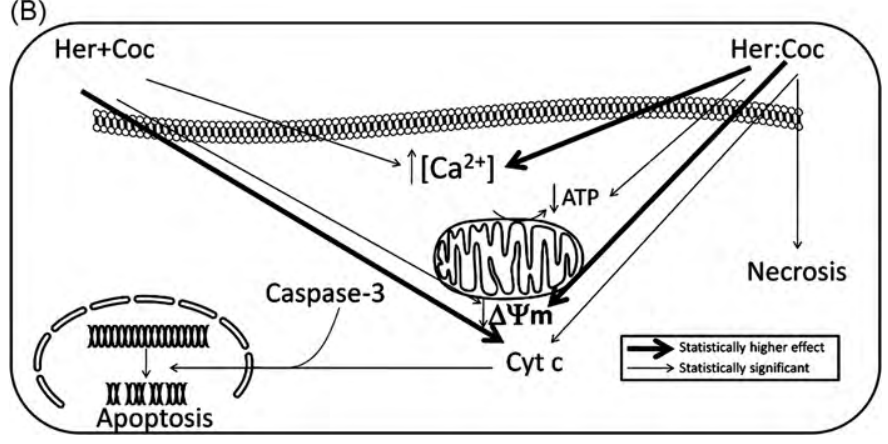

Fig. 5. Effects of heroin ( $1 \mathrm{mM}, 24 \mathrm{~h})$, cocaine $(1 \mathrm{mM}, 24 \mathrm{~h}$ ) or their $1: 1$ combinations on intracellular pathways of neurotoxicity in rat cortical neurons. (A) Heroin and cocaine alone decreased mitochondrial potential and increased caspase-3 activity. Cocaine further decreased intracellular ATP and increased intracellular calcium levels. (B) Sequential $(\mathrm{Her}+\mathrm{Coc}$ ) and simultaneous ( $\mathrm{Her}: \mathrm{Coc})$ combinations of heroin and cocaine increased intracellular calcium levels, decreased mitochondrial membrane potential and mitochondrial cytochrome c levels, increased caspase-3 activity and DNA fragmentation, characteristic of apoptotic cell death. Her + Coc induced a larger decrease in mitochondrial cytochrome c levels, compared to Her:Coc. In contrast, Her:Coc induced a larger increase in intracellular calcium concentration and a larger decrease in mitochondrial membrane potential, compared to $\mathrm{Her}+\mathrm{Coc}$, and further induced a decrease in intracellular ATP levels and increased plasma membrane permeability, characteristic of necrotic cell death.

acute intoxication may greatly exceed those in plasma. In a rat model, cocaine concentration in the brain reached 9 times the plasma concentration, upon $15 \mathrm{~min}$ of administration (Chow et al., 1985). In cocaine-poisoning cases, in humans, cocaine brain concentration was approximately 4 times the blood concentration, ranging from 0.7 to 155 times (Spiehler and Reed, 1985). Therefore, the concentrations of cocaine used in the present study are in the range of those found in plasma in cases of cocaine overdose. Since cocaine:morphine adducts form in a 1:1 proportion (Garrido et al., 2007), heroin concentration in our study was the same used for cocaine. However, it is unlikely that the drug levels in the brain of speedball users achieve the concentrations used in the present study.

In the present work, cocaine induced an increase in intracellular calcium concentration and decreases in mitochondrial membrane potential and ATP levels. The results are in agreement with previous studies showing that cocaine $(0.1-10 \mathrm{mM})$ increased cytosolic and mitochondrial calcium concentration and mitochondrial membrane permeabilization in H9C2 heart-derived cells (Lattanzio et al., 2005). Moreover, it was shown that acute administration of cocaine $(1 \mathrm{mg} / \mathrm{kg}$ ) in a rat model (in vivo) increases intracellular calcium concentration in the rat cerebral cortex, independently of dopaminergic or noradrenergic actions, and likely related to cocaine's local anesthetic effects (Du et al., 2006). It was also suggested that cocaine directly binds and facilitates the opening of L-type calcium channels, leading to increased intracellular calcium levels in cardiac myocytes (Premkumar, 1999). Cocaine also increases the responsiveness of voltage-sensitive calcium chan- nels to membrane depolarization in rat medial prefrontal cortex neurons, upon repeated administration (Nasif et al., 2005). We previously showed that cocaine neurotoxicity in rat cortical neurons was dependent on mitochondrial dysfunction, which seemed to be partially mediated by interactions with the mitochondrial electron transport chain (Cunha-Oliveira et al., 2006). This could explain the decrease in ATP levels observed upon exposure to cocaine or the mixture of cocaine and heroin (Her:Coc). Cocaine (1 mM) also decreased mitochondrial potential and intracellular ATP levels in cardiomyocytes (Yuan and Acosta, 1996).

Cocaine neurotoxicity may also be mediated by a decrease in Bcl-2/Bax in PC12 cells (Lepsch et al., 2009), in locus coeruleus noradrenergic neurons (Dey et al., 2007) and in fetal rat brain (Xiao and Zhang, 2008). In rat cortical neurons, we have previously shown that cocaine induced apoptosis associated with a decrease in mitochondrial membrane potential and an increase in the activity of caspases-2, -9 and -3 (Cunha-Oliveira et al., 2006). Moreover, street heroin neurotoxicity in cortical neurons involved mitochondrialdependent apoptotic pathway, cytochrome c release, loss of mitochondrial membrane potential, caspase-3 activation and down-regulation of Bcl-2 (Cunha-Oliveira et al., 2007). Thus, changes in Bcl-2/Bax could explain the decrease in mitochondrial levels of cytochrome c, and the subsequent apoptotic process, observed upon exposure to both drug combinations, especially $\mathrm{Her}+\mathrm{Coc}$. In the case of Her:Coc, the increase in intracellular calcium concentration, coupled with a decrease in ATP levels and a decrease in mitochondrial potential, also suggest the activation of the mitochondrial transition pore (PTP), which was previously shown to be involved both in apoptotic and necrotic processes (Orrenius et al., 2003). Since upon Her:Coc exposure all of these parameters are increased, when compared to Her + Coc, the opening of the PTP may explain the increase in PI-positive cells upon exposure to Her:Coc. Moreover, the decrease in intracellular ATP levels in Her:Coc may be associated with the increase in necrotic cells, since ATP is needed for the apoptotic process. In addition, a possible decrease in Bcl-2 levels could help to explain the increase in intracellular calcium concentration upon cocaine or Her:Coc exposures, since Bcl-2 was shown to regulate calcium concentration at the endoplasmic reticulum (Pinton and Rizzuto, 2006).

The results presented here suggest that the simultaneous combination of drugs (Her:Coc) maintains the toxicological properties from cocaine, since both Coc and Her:Coc lead to increased intracellular calcium concentration and decreased intracellular ATP levels (Fig. 5). However, Her:Coc induces a higher level of cell death, mediated by a larger decrease in mitochondrial membrane potential and increased intracellular calcium levels, showing both apoptotic and necrotic characteristics. This may be due to the presence of cocaine-morphine adducts, which may affect the interaction with opioid receptors, since the 3-OH of morphine is structurally unavailable in the adduct form (Garrido et al., 2007). This 3$\mathrm{OH}$ group is important for the interaction of morphine with the opioid receptor, as suggested by the fact that heroin has to be previously deacetylated in order to exert effects on brain opioid receptors (Inturrisi et al., 1983; Selley et al., 2001). Another difference between Her + Coc and Her:Coc exposures is that in Her + Coc heroin, or its metabolites, have access to the cells before cocaine does, and thus may affect the way the cells respond to cocaine afterwards. These results clearly show that the mode of co-use of cocaine and heroin impacts on the neurotoxicity of these two drugs, which are definitively more toxic when combined than when administered separately. Chemical interactions between cocaine, heroin and their metabolites, such as the formation of cocaine-morphine adducts, are suggested to shift cell death mechanisms towards necrosis.

Although the complete mechanisms of action of cocaine-heroin combinations have yet to be described, these results show for the 
first time that cocaine and heroin, in the same amounts, have different effects depending on the mode of co-exposure, and suggest that poly-drug abusers are more prone to develop neurotoxic damage than single drug abusers. Since impairment of the prefrontal cortex is involved in the loss of impulse control observed in drug addicts (Volkow et al., 2003), the data presented here may contribute to explain the increase in treatment failure observed in speedball abusers.

\section{Conflict of interest statement}

None.

\section{Acknowledgements}

Financed by FCT- Project POCI/SAU-FCF/58330/2004- and Institute for Interdisciplinary Research, University of Coimbra - Project III/34/2008. TCO is a post-doctoral fellow (SFRH/BPD/34711/2007).

\section{References}

Andersen, J.M., Ripel, A., Boix, F., Normann, P.T., Morland, J., 2009. Increased locomotor activity induced by heroin in mice: pharmacokinetic demonstration of heroin acting as a prodrug for the mediator 6-monoacetylmorphine in vivo. J. Pharmacol. Exp. Ther. 331, 153-161.

Bandettini di Poggio, A., Fornai, F., Paparelli, A., Pacini, M., Perugi, G., Maremmani, I., 2006. Comparison between heroin and heroin-cocaine polyabusers: a psychopathological study. Ann. N. Y. Acad. Sci. 1074, 438-445.

Chow, M.J., Ambre, J.J., Ruo, T.I., Atkinson Jr., A.J., Bowsher, D.J., Fischman, M.W., 1985. Kinetics of cocaine distribution, elimination, and chronotropic effects. Clin. Pharmacol. Ther. 38, 318-324.

Cunha-Oliveira, T., Rego, A.C., Cardoso, S.M., Borges, F., Swerdlow, R.H., Macedo, T., de Oliveira, C.R., 2006. Mitochondrial dysfunction and caspase activation in rat cortical neurons treated with cocaine or amphetamine. Brain Res. 1089, 44-54.

Cunha-Oliveira, T., Rego, A.C., Garrido, J., Borges, F., Macedo, T., Oliveira, C.R., 2007. Street heroin induces mitochondrial dysfunction and apoptosis in rat cortical neurons. J. Neurochem. 101, 543-554.

Dey, S., Mactutus, C.F., Booze, R.M., Snow, D.M., 2007. Cocaine exposure in vitro induces apoptosis in fetal locus coeruleus neurons by altering the Bax/Bcl-2 ratio and through caspase-3 apoptotic signaling. Neuroscience 144, 509-521.

Du, C., Yu, M., Volkow, N.D., Koretsky, A.P., Fowler, J.S., Benveniste, H., 2006. Cocaine increases the intracellular calcium concentration in brain independently of its cerebrovascular effects. J. Neurosci. 26, 11522-11531.

EMCDDA, 2008. Annual Report 2008: the state of the drugs problem in Europe. http://www.emcdda.europa.eu/attachements.cfm/att_64227_EN_EMCDDA_ AR08_en.pdf.

EMCDDA, 2009. Polydrug use: patterns and responses. http://www.emcdda. europa.eu/attachements.cfm/att_93217_EN_EMCDDA_SI09_polydrug\%20use.pdf.

Garrido, J.M., Marques, M.P., Silva, A.M., Macedo, T.R., Oliveira-Brett, A.M., Borges, F., 2007. Spectroscopic and electrochemical studies of cocaine-opioid interactions. Anal. Bioanal. Chem. 388, 1799-1808.

Garrido, J.M.P.., Delerue-Matos, C., Borges, F., Macedo, T.R.A., Oliveira-Brett, A.M., 2004. Voltammetric oxidation of drugs of abuse III. Heroin and metabolites. Electroanalysis 16, 1497-1502.
Goldberger, B.A., Cone, E.J., Grant, T.M., Caplan, Y.H., Levine, B.S., Smialek, J.E., 1994. Disposition of heroin and its metabolites in heroin-related deaths. J. Anal. Toxicol. 18, 22-28.

Guillot, E., de, M.P., Durigon, M., Alvarez, J.C., 2007. Morphine and 6-acetylmorphine concentrations in blood, brain, spinal cord, bone marrow and bone after lethal acute or chronic diacetylmorphine administration to mice. Forensic Sci. Int. 166 139-144.

Heard, K., Palmer, R., Zahniser, N.R., 2008. Mechanisms of acute cocaine toxicity. Open Pharmacol. J. 2, 70-78.

Inturrisi, C.E., Schultz, M., Shin, S., Umans, J.G., Angel, L., Simon, E.J., 1983. Evidence from opiate binding studies that heroin acts through its metabolites. Life Sci. 33 (Suppl. 1), 773-776.

Lattanzio Jr., F.A., Tiangco, D., Osgood, C., Beebe, S., Kerry, J., Hargrave, B.Y., 2005. Cocaine increases intracellular calcium and reactive oxygen species, depolarizes mitochondria, and activates genes associated with heart failure and remodeling. Cardiovasc. Toxicol. 5, 377-390.

Lee, M.C., Cahill, C.M., Vincent, J.P., Beaudet, A., 2002. Internalization and trafficking of opioid receptor ligands in rat cortical neurons. Synapse 43, 102-111.

Lepsch, L.B., Munhoz, C.D., Kawamoto, E.M., Yshii, L.M., Lima, L.S., Curi-Boaventura, M.F., Salgado, T.M., Curi, R., Planeta, C.S., Scavone, C., 2009. Cocaine induces cell death and activates the transcription nuclear factor kappa-b in pc12 cells. Mol. Brain 2, 3 .

Leri, F., Bruneau, J., Stewart, J., 2003. Understanding polydrug use: review of heroin and cocaine co-use. Addiction 98, 7-22.

Nasif, F.J., Hu, X.T., White, F.J., 2005. Repeated cocaine administration increases voltage-sensitive calcium currents in response to membrane depolarization in medial prefrontal cortex pyramidal neurons. J. Neurosci. 25, 3674-3679.

Nutt, D., King, L.A., Saulsbury, W., Blakemore, C., 2007. Development of a rational scale to assess the harm of drugs of potential misuse. Lancet 369, 1047-1053.

Oliveira, M.T., Rego, A.C., Morgadinho, M.T., Macedo, T.R., Oliveira, C.R., 2002. Toxic effects of opioid and stimulant drugs on undifferentiated PC12 cells. Ann. N. Y. Acad. Sci. 965, 487-496.

Orrenius, S., Zhivotovsky, B., Nicotera, P., 2003. Regulation of cell death: the calciumapoptosis link. Nat. Rev. Mol. Cell Biol. 4, 552-565.

Pinton, P., Rizzuto, R., 2006. Bcl-2 and $\mathrm{Ca}^{2+}$ homeostasis in the endoplasmic reticulum. Cell Death Differ. 13, 1409-1418.

Premkumar, L.S., 1999. Selective potentiation of L-type calcium channel currents by cocaine in cardiac myocytes. Mol. Pharmacol. 56, 1138-1142.

Rook, E.J., Huitema, A.D., van den Brink, W., van Ree, J.M., Beijnen, J.H., 2006. Pharmacokinetics and pharmacokinetic variability of heroin and its metabolites: review of the literature. Curr. Clin. Pharmacol. 1, 109-118.

Selley, D.E., Cao, C.C., Sexton, T., Schwegel, J.A., Martin, T.J., Childers, S.R., 2001 mu Opioid receptor-mediated G-protein activation by heroin metabolites: evidence for greater efficacy of 6-monoacetylmorphine compared with morphine. Biochem. Pharmacol. 62, 447-455.

Spiehler, V.R., Reed, D., 1985. Brain concentrations of cocaine and benzoylecgonine in fatal cases. J. Forensic Sci. 30, 1003-1011.

Strandberg, J.J., Kugelberg, F.C., Alkass, K., Gustavsson, A., Zahlsen, K., Spigset, O., Druid, H., 2006. Toxicological analysis in rats subjected to heroin and morphine overdose. Toxicol. Lett. 166, 11-18.

Volkow, N.D., Fowler, J.S., Wang, G.J., 2003. The addicted human brain: insights from imaging studies. J. Clin. Invest. 111, 1444-1451.

Xiao, D., Zhang, L., 2008. Upregulation of Bax and Bcl-2 following prenatal cocaine exposure induces apoptosis in fetal rat brain. Int. J. Med. Sci. 5, 295-302.

Yuan, C., Acosta Jr., D., 1996. Cocaine-induced mitochondrial dysfunction in primary cultures of rat cardiomyocytes. Toxicology 112, 1-10.

Yuan, C., Acosta Jr., D., 2000. Effect of cocaine on mitochondrial electron transport chain evaluated in primary cultures of neonatal rat myocardial cells and in isolated mitochondrial preparations. Drug Chem. Toxicol. 23, 339348. 